\author{
J. A. Pomarico · H. O. Di Rocco · L. Alvarez \\ C. Lanusse · L. Mottier · C. Saumell · R. Arizaga \\ H. Rabal $\cdot$ M. Trivi
}

\title{
Speckle interferometry applied to pharmacodynamic studies: evaluation of parasite motility
}

Received: 17 December 2003/ Accepted: 18 April 2004/Published online: 7 May 2004

(c) EBSA 2004

\begin{abstract}
The work reported here describes the application of the optical technique known as dynamic speckle interferometry to evaluate the motility of nematode parasites exposed to different anthelmintic drugs. This technique, a well proven tool for assessing the time evolution of different phenomena, is here successfully used to quantify parasite motility in pharmacodynamic assays. The characterization of the pharmacological properties of anthelmintic drugs is critical to optimize their use in parasite control. Besides, the evaluation of nematode motility is a relevant indicator of the pharmacodynamic effect of anthelmintic drugs. The application of this approach to study the motility of Haemonchus contortus (used as a model of nematode parasites) larvae exposed to different drugs is presented, showing its usefulness.
\end{abstract}

Keywords Parasites $\cdot$ Pharmacodynamics $\cdot$ Speckle

Abbreviations ABZ: albendazole - BZD: benzimidazole IVM: ivermectin $\cdot$ LVM: levamisole

\section{Introduction}

Chemotherapy is the most widely used method to control, prevent and treat parasitism. In order to optimize the clinical effectiveness of a drug, it is relevant to

J. A. Pomarico $(\varangle) \cdot$ H. O. Di Rocco

Instituto de Física Arroyo Seco (IFAS),

Facultad de Ciencias Exactas, UNCPBA,

Pinto 399, B7000GHGTandil, Argentina

E-mail: juanp@exa.unicen.edu.ar

L. Alvarez $\cdot$ C. Lanusse $\cdot$ L. Mottier $\cdot$ C. Saumell

Facultad de Ciencias Veterinarias, UNCPBA,

Pinto 399, B7000GHG Tandil, Argentina

R. Arizaga $\cdot$ H. Rabal $\cdot$ M. Trivi

Centro de Investigaciones Ópticas (CIC-CONICET)

and UID Optimo, Departamento de Fisicomatemáticas,

Facultdad de Ingeniería UNLP, 1900 La Plata, Argentina understand the pharmacological principles that contribute to the final activity, including pharmacokinetic and pharmacodynamic data. A rational approach to, for example, anthelmintic therapy requires a knowledge of the drug concentrations at the site of infection and of the minimum inhibitory concentrations (MIC) against parasitic stages of the nematode (Baggot and McKellar 1994).

The activity of most anthelmintic molecules is based on their affinity for a specific receptor and on the kinetic properties that allow the delivery of effective drug concentrations to the receptor inside the parasite, in sufficient time to cause the therapeutic effect (Thompson et al. 1993). Understanding the concentration-timeantiparasitic effect relationship is crucial for the rational use of anthelmintics. Because of cost difficulties derived from in vivo experiments, in vitro tests could be a useful tool toward this end. The development of in vitro tests would contribute to investigations of the relationship between the anthelmintic effect and potency of different drugs over adult/larval nematode parasites, the detection of parasite resistance (and its degree) and to find potential new anthelmintic molecules. However, the main disadvantage of most of the in vitro tests developed that evaluate parasite motility are their subjectivity, e.g. the larval paralysis test described by Martin and Le Jambre (1979), or that they evaluate parasite motility using adult worms, so it is necessary to sacrifice the infected animals.

In the present experimental work, we describe a novel physiologically based assay methodology for the in vitro testing of parasite motility. The methodology is based on an optical technique known as dynamic speckle interferometry, often called biospeckle interferometry (Aizu and Asakura 1996). The basic concepts of the technique are described and applied to several samples, following the time evolution of the activity of dynamic speckle patterns. Haemonchus contortus (third-stage larvae, L3) incubated with albendazole (ABZ), ivermectin (IVM) and levamisole (LVM) was used as an experimental model. 
In the following section we present a short background of speckle fundamentals and the way of processing the data. For more details about speckle phenomenon, the reader is invited to look at the several specialized books on the matter (e.g. Dainty 1975; Sirohi 1993). Next, a description is given concerning the materials, methods and experimental procedures of the work. Finally, some results are presented. The good agreement of these with other well-established methods shows the usefulness and applicability of the proposed technique.

Fundaments of speckle interferometry

When an optically rough surface is illuminated by laser light it exhibits a particular granular appearance named laser speckle (Dainty 1975; Sirohi 1993). This is due to the interference of coherent wavelets coming from different points of the rough object. The distances travelled by these various wavelets may differ by several or many wavelengths if the surface is truly rough. The detailed structure of this granularity is random (since the surface topography is also random) and is best described by the methods of probability theory and statistics. The same concepts apply when transilluminating a rough transparent medium, like a diffuser (ground glass). In this case the micro curvatures of the glass surface act as a set of positive and negative lenses, randomly located and with a random distribution of focal lengths. As a result, multiple coherent de-phased spherical wavelets emerge from the diffuser and their interference in the observation plane gives rise to a speckle pattern. This situation is schematically represented in Fig. 1, illustrating the physical origin of the speckle phenomenon. A photograph of a typical speckle pattern is also shown.

Additionally, when laser light is scattered by objects showing some type of activity (fruits and other bio- logical samples), the visual appearance of the pattern is similar to that of a boiling liquid. It is called dynamic speckle or biospeckle. This effect is due to variations in the phase of the light produced by movements of the scatterers, changes in the refractive index, rotatory power, etc. Light coming from different scatterers produces low-frequency beats in the detector that appear as variations in local intensity. This phenomenon has been used to measure the dynamic activity of seeds (Arizaga et al. 2002), bruising in fruits (Pajuelo et al. 2003), etc. The remaining task is to assign a numerical value to the speckle activity, thus quantifying the biological activity of the sample. This will be the subject of the Results and discussion section.

\section{Materials and methods}

Test compounds

Pure reference standards of ABZ (Schering Plough, Kenilworth, USA), IVM and LVM (Sigma-Aldrich, Steinheim, Germany) were used in the experimental work.

\section{Parasites}

The third-stage larvae (L3) of Haemonchus contortus used in this study were obtained from a farm with little or no exposure to any anthelmintic. The isolates were routinely maintained by passage to 6- to 8-month-old worm-free Corriedale sheep. L3 larvae were recovered from faecal cultures by standard Baermann filtration and stored at $10{ }^{\circ} \mathrm{C}$ in tap-water prior to use. All L3 larvae used in the tests were $\leq 1$ month old at the time of testing.
Fig. 1 Schematic

representation of the physical principle behind the origin of speckle patterns. A photograph of an actual pattern, obtained by using a CCD camera at the observation plane, is included

\section{Physical Origin of Speckle}

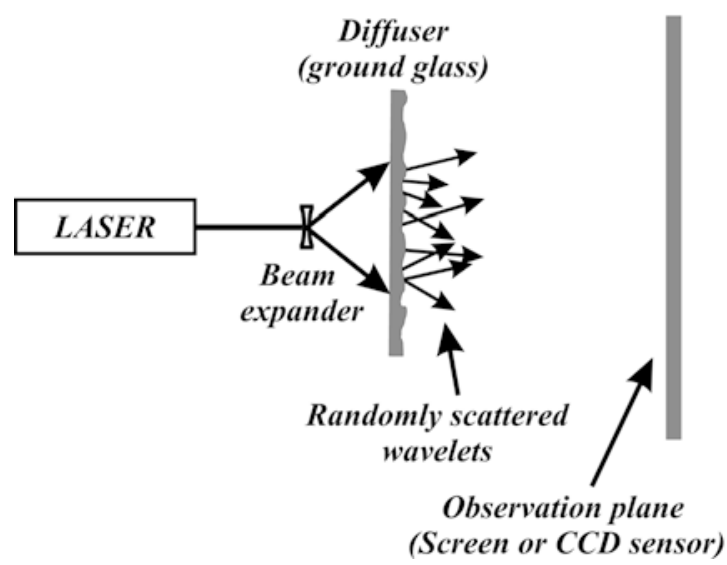

Actual speckle diagram

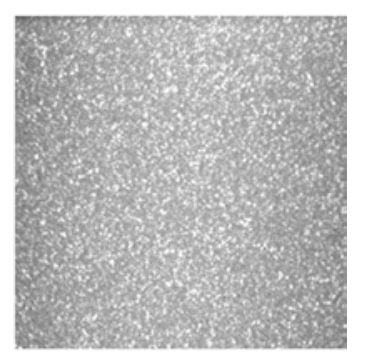


In vitro assay procedures

\section{Effect of L3 concentration}

The capacity of the method to discriminate different larvae concentrations was tested by analyzing the following L3 concentrations: 100, 200, 400, 600, 800, 1200 and $1600 \mathrm{~mL}^{-1}$ of Kreb's Ringer Tris (KRT) buffer. Aliquots $(250 \mu \mathrm{L})$ of each concentration were placed in special plates and maintained at $20^{\circ} \mathrm{C}$ during the analytical procedures. The quantification of each larval concentration was made by direct optical count using a standard optical microscope (Nikon, Japan, 100×).

\section{Drug effect on L3 motility}

The capability of the method to measure the effect of the different drugs assayed on larval motility was tested. One milliliter of a suspension containing 750 larvae was incubated for $72 \mathrm{~h}$ in KRT buffer (pH 7.4) at $20^{\circ} \mathrm{C}$ containing either ABZ, IVM or LVM. There were four $(n=4)$ replicates at a final concentration of $1 \mu \mathrm{g} \mathrm{mL}{ }^{-1}$, $5 \mathrm{ng} \mathrm{mL} \mathrm{m}^{-1}$ and $2 \mu \mathrm{g} \mathrm{mL}^{-1}$, respectively. These are pharmacologically relevant concentrations obtained from previously reported studies. Two control groups were incorporated. A positive control with L3 incubated without drug, and a negative control with dead L3 (by $100 \mu \mathrm{L}$ formaldehyde), were incubated during the same time interval.

\section{Assessment of drug incubation time on L3 motility}

One milliliter of a suspension of L3 larvae containing $750 \mathrm{~mL}^{-1}$ was incubated in KRT buffer $(\mathrm{pH} \mathrm{7.4)}$ at $20{ }^{\circ} \mathrm{C}$ containing either ABZ $\left(1 \mu \mathrm{g} \mathrm{mL}^{-1}\right)$, IVM $(5 \mathrm{ng}$ $\left.\mathrm{mL}^{-1}\right)$ or LVM $\left(5 \mu \mathrm{g} \mathrm{mL}^{-1}\right)$ during the following incubation times: $0,18,42,66$ and $90 \mathrm{~h}$. There were four $(n=4)$ replicate assays for each drug. Two control groups were incorporated. Positive and negative controls were incubated during the same time interval.

\section{Experimental}

For registering the activity of a speckle pattern, first a set of successive images of it were recorded. For doing that, we used the experimental setup shown in Fig. 2. An expanded $10 \mathrm{~mW}$ helium-neon red laser $(\lambda=633 \mathrm{~nm})$, attenuated with a neutral density wedge filter, was used to normally illuminate the samples. The parasites were kept in trays containing $0.25 \mathrm{~mL}$ of methanol and the laser was conveniently expanded so as to illuminate the whole bottom of the tray. This was to ensure that all parasites in the sample were illuminated, thus contributing to the activity of the resulting speckle pattern. A diffuser was placed immediately below the tray to produce a good contrast speckle pattern. A defocused CCD camera connected to a PC with a frame grabber was

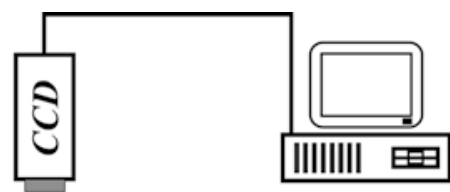

Image processing unit

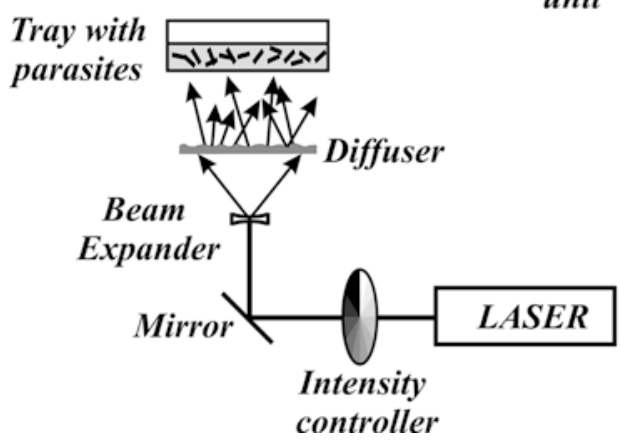

Fig. 2 Experimental setup used to record the dynamic speckle patterns due to parasite activity

used as the recording device to digitize the image. Care was taken that the speckles were well resolved by the CCD sensor. The registers were made in a room with motionless air and a constant temperature to limit the variables that affect the process. The humidity and temperature were those typical of a laboratory: room temperature ranged from 20 to $25^{\circ} \mathrm{C}$ and the humidity from 50 to $70 \% \mathrm{RH}$.

\section{Acquisition and handling of image data}

In this section we describe one way to assign a numerical measure to dynamic speckle patterns. Images were acquired by taking a short movie (typically lasting 1-2 s) of the dynamic speckle pattern produced by the moving parasites according to the experimental setup in Fig. 2. At a rate of 30 frames per second, more than 30 pictures were available per movie. Next, a set of frames (typically 8 ) were downloaded using a frame-by-frame mode in the image processing unit. With the image processor used, the minimum achievable delay between consecutive pictures is $\tau_{\min }=0.033 \mathrm{~s}$. Each frame has an extension of $480 \times 480$ pixels and is coded in eight bits, giving rise to 256 gray level images.

Image correlation (IC) can be used for evaluating the activity of the speckle pattern. In fact, given two full frames (each of them of $480 \times 480$ pixels) of the speckle pattern acquired one after the other at a certain time interval $(\tau)$, it is possible to construct the following correlation function based on the intensity difference:

$D(\tau)=\frac{1}{N} \sum_{i j}|I(i, j, t)-I(i, j, t+\tau)|$

where $I(i, j, t)$ and $I(i, j, t+\tau)$ are the image intensities (gray level) at pixel $(i, j)$ and at times $t$ and $t+\tau$, respectively. 
The time delay between images $(\tau)$ must be properly chosen to avoid undersampling. This means that any fluctuation in speckle intensity should have a characteristic time satisfying $t_{\text {char }}>>\tau$. Note that, necessarily, $\tau \geq \tau_{\min }$, thus setting a limit to the maximum activity that can be properly detected. In Eq. 1, $N$ is a normalization factor, independent of $\tau$, which is usually set to the maximum allowable value for the summation in the definition of $D(\tau)$, that is:

$$
\begin{aligned}
N & =\left[\sum_{i j}|I(i, j, t)-I(i, j, t+\tau)|\right]_{\max } \\
& =\left(I_{\max }-I_{\min }\right) \times 480 \times 480=255 \times 480 \times 480
\end{aligned}
$$

where $I_{\max }$ and $I_{\min }$ are, respectively, the maximum (255) and minimum (0) gray level attainable in eight bits codification. With this choice, $D(\tau)$ results in a number restricted to the interval $[0,1]$.

Clearly, for a given $\tau$, if the activity of the speckle diagram is low, the gray level at any location $(i, j)$ varies slowly with time and thus $I(i, j, t) \approx I(i, j, t+\tau)$. The resulting $D(\tau)$ is accordingly small. On the other hand, for speckle diagrams showing high activity, and irrespective of the location $(i, j), I(i, j, t)$ is significantly different from $I(i, j, t+\tau)$ and so $D(\tau)$ is close to 1 .

An alternative and convenient way to show the time evolution of a speckle pattern is that proposed by Oulamara et al. (1989), based on a technique called time history of the speckle pattern. A certain column (for example, the middle one) in $M$ successive images of the dynamic speckle pattern is registered and stored in a frame grabber. A new image is then constructed composed by setting, side by side, the chosen column along the register time. The resulting synthetic image is named the time history of the speckle pattern (THSP). In it, different points on the object are represented by the different rows and the columns show their intensity state in every time step. The activity of the sample appears as the intensity changes in the horizontal direction.

Some authors (Arizaga et al. 1999) have described a way to obtain a numerical measurement of the activity of speckle patterns starting from the THSP. The proposal makes use of the so-called co-occurrence $(\mathrm{CO})$ matrix (Haberacker 1985) and the activity is quantified in terms of its second-order moment, called the moment of inertia. For a detailed description of this method, the paper by Allam et al. (1997) can be consulted. This measurement is similar to one currently used in photon correlation spectroscopy, called photon structure function (Chu 1991).

Although it is clear that the IC algorithm described above and the THSP evaluated with the $\mathrm{CO}$ matrix do not produce strictly the same numerical result, they are totally equivalent and both approaches give the same amount of information. The results presented in this work correspond to evaluations using the IC algorithm.

\section{Results and discussion}

Activity shown by the speckle pattern becomes greater because of both parasite concentration (every individual organism contributes to the whole activity) and ensemble motility. Because of this, before evaluating any drug action upon the samples, it is important to investigate the influence of parasite concentration on the final numerical value of the correlation function $D(\tau)$, defined in Eq. 1 . Moreover, the dynamic range of the approach must be evaluated, since it is to be expected that (for a given sampling time interval) a very low parasite concentration does not produce any measurable activity of the speckle pattern and too high a concentration leads to a saturation of the measurement due to undersampling effects.

\section{Investigation of L3 concentration}

Figure 3 shows a plot of motility versus L3 concentration. The data adjust very well to a sigmoidal curve, being linear for concentrations between 400 and $1000 \mathrm{~mL}^{-1}$ of L3. For this experiment the time interval between images was set to $\tau=0.033 \mathrm{~s}$ to avoid undersampling. This was checked by varying $\tau$ up to values as great as $0.1 \mathrm{~s}$, where the obtained values of $D(\tau)$ began to fluctuate, thus indicating correlation loss. The plotted points are the result of averaging four values of $D(\tau)$ resulting from pairs of consecutive speckle images for different time values inside the $2 \mathrm{~s}$ movie. In symbols this is :

$$
\begin{aligned}
D_{\mathrm{av}}(\tau) & =\frac{1}{4} \sum_{k=1}^{4} D_{k}(\tau) \\
& =\sum_{k=1}^{4}\left[\frac{1}{4 N} \sum_{i j}\left|I\left(i, j, t_{k}\right)-I\left(i, j, t_{k}+\tau\right)\right|\right]
\end{aligned}
$$

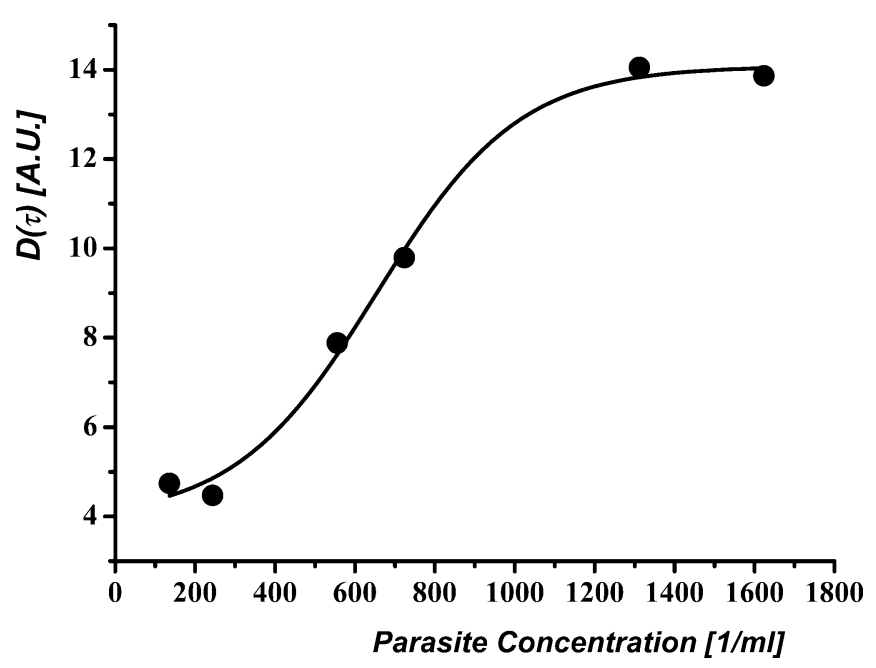

Fig. 3 Representation of the difference function, $D(\tau)$, as a function of the L3 concentration. A clear sigmoidal plot $\left(R^{2}=0.995\right)$ can be observed showing an approximate linear behavior for concentrations between 400 and $1000 \mathrm{~mL}^{-1}$ 


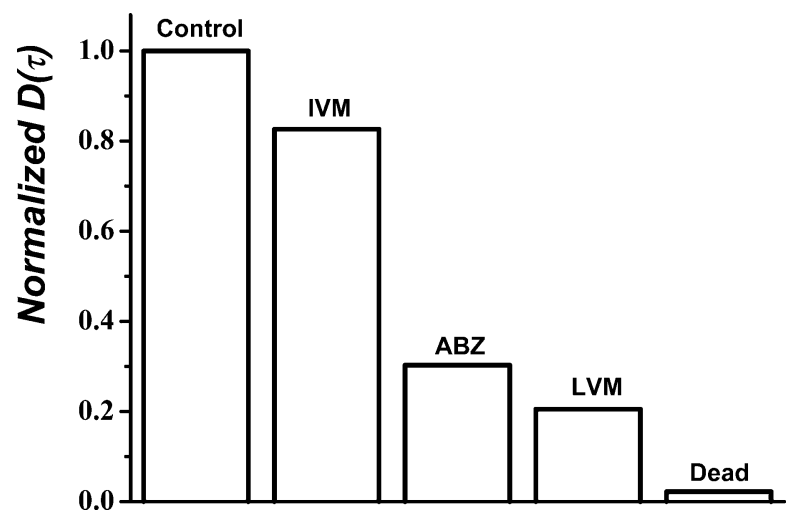

Fig. 4 Quantitative measurement of the L3 activity by means of the difference function. Values are normalized with respect to the control sample and the incubation time was $72 \mathrm{~h}$ for all drugs. The action of the different drugs can be clearly distinguished. The low noise level, of about $2 \%$, gives the resolution limit of the method

where the different $t_{k}$ were uniformly distributed in $0<t_{k}<2$ s.

Investigation of drug incubation time and drug kinetics

A time-dependent effect of the three drugs assayed on L3 motility was verified. As a first experiment we investigated the discrimination capability of the method between different drugs. To this end, three samples were prepared following the procedure described in Materials and methods and incubated for $72 \mathrm{~h}$ in LVM, IVM and $\mathrm{ABZ}$ and the corresponding correlation functions $D(\tau)$ were obtained using a video rate of 10 frames per second. Additionally, a control sample and a sample containing dead larvae were also measured. The motility of the control larvae was assumed to be $100 \%$ and the resulting value of $D(\tau)$ for dead larvae is a measure of the minimum detectability of the method, a threshold set by electronic and/or optical noise. These results are summarized in Fig. 4 and are in qualitative agreement with established results (Prichard et al. 2003).

Next, due to these promising results, we investigated the effect of these drugs as a function of time. For this experiment, the video rate was set to 30 frames per second. The effect of LMV on larval motility was fast and sustained over time. However, a slower effect on larval motility was observed for both ABZ and IVM, reaching a maximum after $72 \mathrm{~h}$ of incubation. Data for larval motility are shown in Fig. 5. The values of $D(\tau)$ for each molecule and each incubation time were analyzed by standard statistical methods. Generally speaking, the relative percent deviations lie between $10 \%$ and $25 \%$. This is depicted in Fig. 5 by the corresponding error bars.

\section{Conclusions}

As concluding remarks it can be said that anthelmintic activity is related to both the duration of parasite

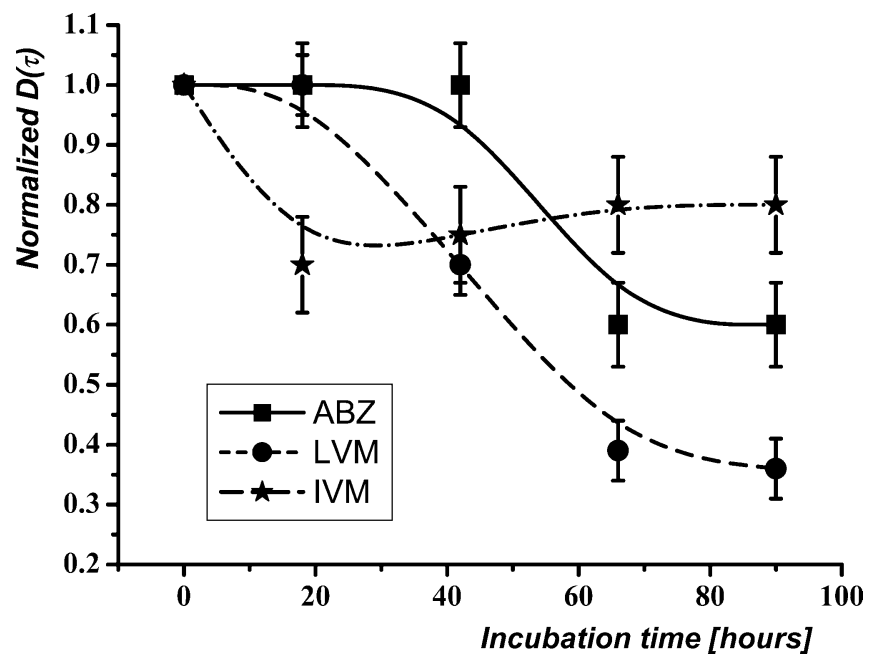

Fig. 5 Normalized time evolution of drug activity. Note that the lines do not represent any fitting, but are just a guide to the eye to help following the corresponding points

exposure to effective concentrations of the active compound at the parasite location site and the action of the drug. There are two major modes of action of anthelmintics (see Martin 1997). There are the drugs that act on parasite membrane ion-channels and which usually have a more rapid therapeutic effect; the other group acts more slowly on a range of "biochemical" target sites found in parasites. The target ion-channels include the ITZ and AVM groups. These two groups open ionchannels, which generates a current across cell membranes of the nematodes, leading to a spastic or flaccid muscular paralysis. ITZ molecules are nicotinic agonists that selectively gate acetylcholine cation channels in somatic muscle of nematodes to produce contraction, paralysis and elimination from the host. The mode of action of AVM is believed to involve the gating (opening) of glutamate-gated chloride channels that are unique to invertebrates, including nematodes and insects. The benzimidazole (BZD) chemical family belongs to the group of drugs that act on biochemical sites. BZD acts by binding to parasite tubulin, thus inhibiting polymerization into microtubules. When the assembly of microtubules is compromised, the nematode cannot sustain biochemical and metabolic homeostasis, so the effects of BZD on helminth parasites are essentially long term and translated into starvation of the nematode by intestinal disruption, inhibition of egg production and an impairment of motility. The onset of BZD anthelmintic action is therefore slower than that of anthelmintics that work directly on ion channels.

The development of drug resistance in parasitic nematode populations has placed limitations on the continued use of many anthelmintics. However, responsible use of new drugs should minimize the development of resistance and prolong their useful life (Walley 1966). Techniques for the detection of resistance are necessary in order to monitor the extent and devel- 
opment of the problem in parasite populations and thus provide a database from which rational control programs can be developed.

Several different tests have been used for the detection of anthelmintic resistance in nematodes of veterinary importance, but few of them have been developed for evaluating parasite motility suitable for the detection of drug resistance and, also, with the purpose of proving or comparing the pharmacological effects of different drugs. The main disadvantages of most of the in vitro tests developed that evaluate parasite motility are their subjectivity, e.g. the larval paralysis test described by Martin and Le Jambre (Boersema 1983; Martin and Le Jambre 1979), or that they evaluate parasite motility using adult worms (Petersen et al. 1997), so it is necessary to sacrifice the infected animals.

The in vitro results obtained in the present experimental work are in agreement with the proposed mechanisms of action and also with in vivo studies, where the importance has been shown of a long duration of exposure to benzimidazoles to achieve optimal efficacy of the drugs (Prichard et al. 2003).

The results reported here demonstrate that the application of dynamic speckle is a novel methodology that evaluates parasite motility. The main advantages of this technique are based on its objectivity, sensitivity and fast recording and processing of the data obtained. Since a method for determining the minimum concentration of a drug that kills or inhibits parasite motility has still not been developed for helminth parasites, this methodology would be used for this proposal. Additionally, a comparison of the power of several anthelmintics, as well as the precocious detection of resistance, could be a future possible application of the described technique.

Acknowledgements This work was partially supported by UNCPBA, UNLP, CONICET, CICPBA and ANPCYT, Argentina.

\section{References}

Allam BS, Adel M, Refregier P (1997) Fast algorithm for texture discrimination by use a separable orthonormal decomposition of the co-occurrence matrix. Appl Opt 36:8313-8321
Arizaga R, Trivi M, Rabal HJ (1999) Speckle time evolution characterization by the co-occurrence matrix analysis. Opt Laser Techn 31:163-169

Arizaga R, Cap N, Rabal HJ, Trivi M (2002) Display of local activity using dynamical speckle patterns. Opt Eng 41:287294

Aizu Y, Asakura T (1996) Biospeckles. In: Consortini A (ed) Trends in optics. Academic Press, London, pp 27-49

Baggot D, McKellar Q (1994) The absorption, distribution and elimination of anthelmintics drugs: the role of phamacokinetics. J Vet Pharmacol Ther 17:409-419

Boersema J (1983) Possibilities and limitations in the detection of anthelmintic resistance. In: Borgsteede F, Henriksen S, Over $\mathrm{H}$ (eds) Facts and reflections. IV. Resistance to anthelmintics. Central Veterinary Institute, The Netherlands, pp 207-216

Chu A (1991) Laser light scattering: basic principles and practice. Academic Press, Boston

Dainty JC (ed) (1975) Laser speckle and related phenomena. Springer, Berlin Heidelberg New York

Haberacker P (1985) Digitale Bildverarbeitung Grundlagen und Anwendungen. Hanser, Munich

Martin P (1997) Modes of action of anthelmintic drugs. Vet J 154:11-34

Martin P, Lejambre L (1979) Larval paralysis as an in vitro assay of levamisole and morantel tartrate resistance in Ostertagia. Vet Sci Commun 3:159-164

Oulamara A, Tribillon G, Duvernoy J (1989) Biological activity measurement on botanical specimens' surfaces using a temporal decorrelation effect of laser speckle. J Mod Opt 36:165179

Pajuelo M, Baldwin G, Rabal H, Cap N, Arizaga R, Trivi M (2003) Bio-speckle assessment of bruising in fruits. Opt Laser Eng 40:13-24

Petersen M, Friis C, Bjørn H (1997) A new in vitro assay of benzimidazole activity against adult Oesophagostomum dentatum. Int J Parasitol 27:1333-1339

Prichard R, Forrester S, Njue X, Feng X, Liu J, Beech R (2003) Receptor mechanisms of antiparasitics. J Vet Pharmacol Ther 26:19-55

Sirohi RS (ed) (1993) Speckle metrology. Dekker, New York

Thompson D, Ho N, Sims S, Geary T (1993) Mechanistic approaches to quantitate anthelmintic absorption by gastrointestinal nematodes. Parasitol Today 9:31-35

Walley J (1966) Tetramisole (dl-2,3,5,6-tetrahydro-6-phenyl-imidazo-[2,1-b]-thiazole hydrochloride, Nilverm) in the treatment of gastro-intestinal worms and lungworms in domestic animals. 1. Sheep and goats. Vet Rec 78:406-414 